\title{
Aircraft Maintenance Professionals: Stress, Pressure and Fatigue
}

\author{
Nicole G. Dias ${ }^{1}$, Luis F.F.M. Santos ${ }^{2}$, and Rui Melicio , $^{2,4, *}$ \\ ${ }^{1}$ Universidade da Beira Interior, Covilhã, Portugal \\ ${ }^{2}$ IDMEC, Instituto Superior Técnico, Universidade de Lisboa, Lisbon, Portugal \\ ${ }^{3}$ ICT, Instituto de Ciências da Terra, Universidade de Évora, Portugal \\ ${ }^{4}$ Departmento de Física, Escola de Ciências e Tecnologia, Universidade de Évora, Évora, Portugal
}

\begin{abstract}
The objective is to emphasize on the environmental and organizational pressure, namely, the pressure and fatigue induced in aircraft professionals. Safety is the main driver of aviation; environmental and organizational conditions, including extended working hours, have a negative impact on the performance of aircraft maintenance professionals and on aircraft safety. Just as aircraft crews and air traffic controllers have their work schedules highly monitored by aviation regulators; but regulators do not monitor the work schedules of professionals and they are subject to extended working timetables required by aeronautic players. The authors, through an international survey aimed at aeronautical professionals, demonstrate that errors are linked to stress, pressure and fatigue, environmental and organizational. This work also intends to demonstrate the need for regulators to monitor the work schedules of aircraft professionals to avoid that extended working hours having a negative impact on aircraft safety. The authors give the possible diagnostics and recommendations, which can be used to help the aeronautical players and aircraft maintenance staffs to create a safety culture and a decision and validation matrix of the aircraft maintenance.
\end{abstract}

\section{Introduction}

Air transport is considered to be one the safest way of transportation [1,2], due to the close interaction between aeronautical regulators and industry, namely operators and maintenance [3]. This interaction allowed having technically more reliable aircraft, reducing accidents/incidents to residual values [4]. But, on the other hand, the accidents/incidents related to human factors have been increasing over time [4]. The operational and maintenance procedures have become highly regulated and highly complex with many interactions between different working groups, that requires sustained coordination, communication and cooperation in order to ensure safety and efficient operations (minimizing error) [5]. Implementing complex operation and maintenance procedures requires the development of innovative solutions that increase efficiency without compromising safety and quality [5], particularly those related to human factors.

\footnotetext{
*Corresponding author: ruimelicio@gmail.com
} 
One way of minimizing errors deriving from stress and fatigue, contributing then to the increased aviation safety, is paying more attention to the working and rest schedules of the professionals involved in aircraft maintenance, as already done with the flight crew [6,7]. In [8] is presented a method for evaluating the pilots mental distress and its effect on the eye pupils, which can be somehow extrapolated to the maintenance professionals.

A detailed description of the current regulatory system is available in [7]. The first set of standards to improve flight safety was introduced by ICAO and led the industry to develop methods to eliminate common causes of accidents/incidents. The second set of standards, also developed by ICAO, was the introduction of regulations to eliminate the multiple and random causes of safety issues, which gave birth to the Safety Management System. One of the major safety increments in aviation was due to ICAO Annex 19 implementation, this implementation was the main driven for human factors implementation [9] into the regulatory system. Exists an entire new research field dedicated only to study of human factors. In fact, these studies are so important that EASA, FAA and ICAO have dedicated teams focused only in the Human Factors $[9,10]$.

The Annex 19, Safety Management System, issued by ICAO, led the worldwide aerospace industries to new safety policies, which were allocated into two major phases [9], Phase 1: Eliminating the Common Causes, and Phase 2: Eliminating the Random/Multiple Causes. In Phase 1 the regulators are concerned about the Technology Improvements, Human Factors and Organizational Factors; and in Phase 2 the focus are the Safety Management Policies and the State Safety Program.

The contribution of this paper is that the authors, through an international research directed to aeronautical maintenance professionals, intend to demonstrate that the errors are linked to stress, pressure and fatigue, environmental and organizational factors. When we look into the Fatigue Risk Management, EASA has created very strict limitations of duty service for aircrews [7,9,11], nevertheless, and regarding the maintenance professionals, both EASA Part-M and Part-145 does not refer any workload limit for these aircraft maintenance professionals $[7,12,13]$. Since the regulator did not create any soft law to instruct about the workload limitations, delegating to the maintenance providers "Maintenance Organization Manuals" [12] or "Continuing Airworthiness Management Exposition Manuals" this responsibility. Since the companies are interested to have $24 \mathrm{~h} / 7$ days available maintenance staff, they create in their manuals these need for the staff availability, putting into stress, pressure and fatigue their staff.

The paper is structured as follows: Section 2 presents the current regulatory system. Section 3 presents the case study. Section 4 presents hazard procedures. Section 5 presents the diagnostic and recommendations. Finally, Section 6 outlines the conclusion.

\section{The need for regulations}

A detailed explanation detailing the need for regulations is available in [7]. There are key indicators which demands regulations on maintenance professionals. As can be seen in Section 3, exists indicators that regulators must pay special attention too. The examples presented in [14] determine the need to identify in early stages psychological, stress or fatigue conditions that might lead to hazardous occurrences. After identification, it will allow to prevent and mitigate these hazardous behaviours leading the industry to new higher levels of safety. 


\section{Case study}

An online international survey has been carried out to figure what are the current status of the technical staff regarding pressure, stress and fatigue. The participants were maintenance engineers and technicians, working on Part-145 and Part-M organizations from Portugal, Spain, France, Germany and UK $[15,16]$. A detailed description of the international survey is available in [7].

The survey age sample distribution is shown in Fig. 1.

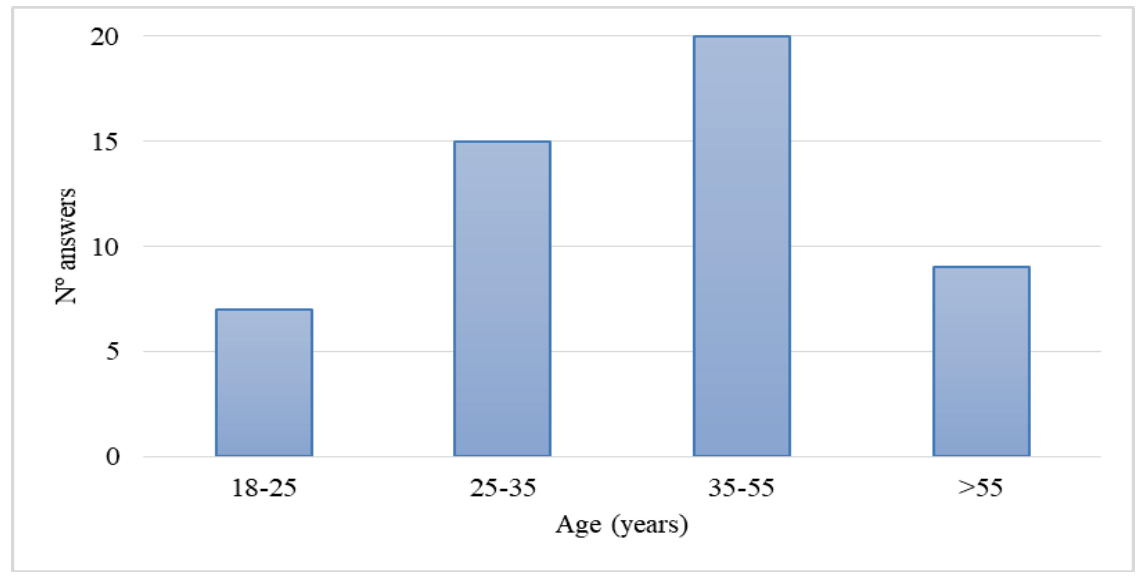

Fig. 1. Survey age sample distribution.

In Fig. 1 the age distribution consists of: $13.73 \%$ of ages between 18 years and 25 years old; $29.41 \%$ of ages between 25 years and 35 years old; $39.22 \%$ between the ages of 35 years and 55 years old; $17.65 \%$ of over 55 years old.

The combination of the very high physical fatigue (blue) and the very high mental fatigue (orange) with the age distribution is shown in Fig. 2.

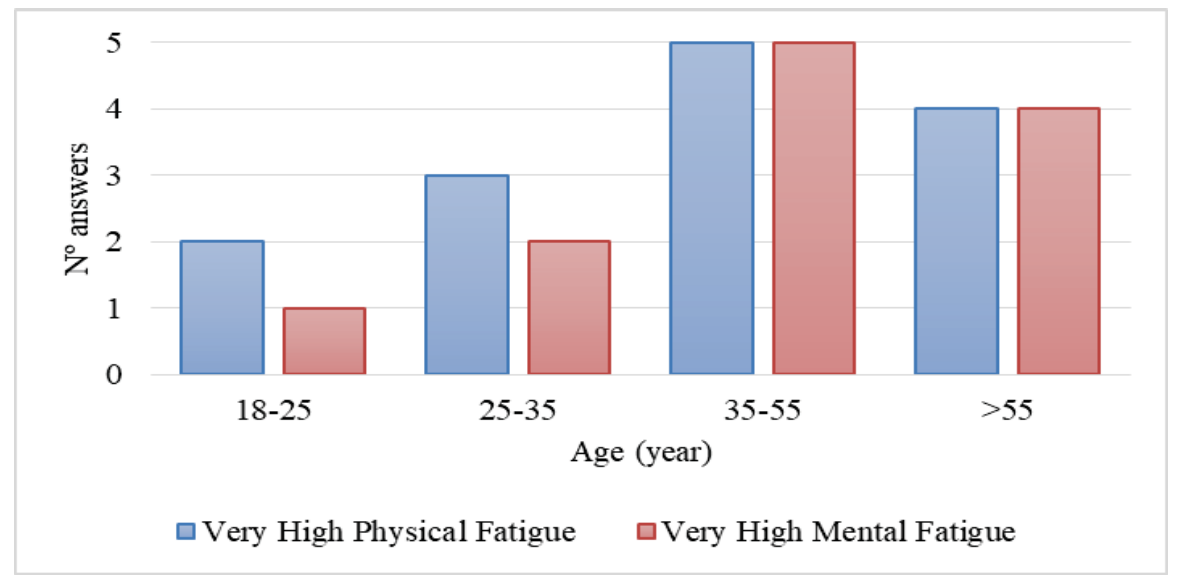

Fig. 2. Very high physical fatigue (blue) and the very high mental fatigue (orange). 
In Fig. 2, 3.92\% of ages between 18 years and 25 years old; $5.88 \%$ of ages between 25 years and 35 years old; $9.80 \%$ between the ages of 35 years and 55 years old; $7.84 \%$ of over 55 years old felt very high physical fatigue (blue); while 1.96\% of ages between 18 years and 25 years old; $3.92 \%$ of ages between 25 years and 35 years old; $9.80 \%$ between the ages of 35 years and 55 years old; $7.84 \%$ of over 55 years old felt very high mental fatigue (orange).

Regarding the question is it considered that the rest times you enjoy are sufficient: yes (blue) and no (orange) are shown in Fig. 3.

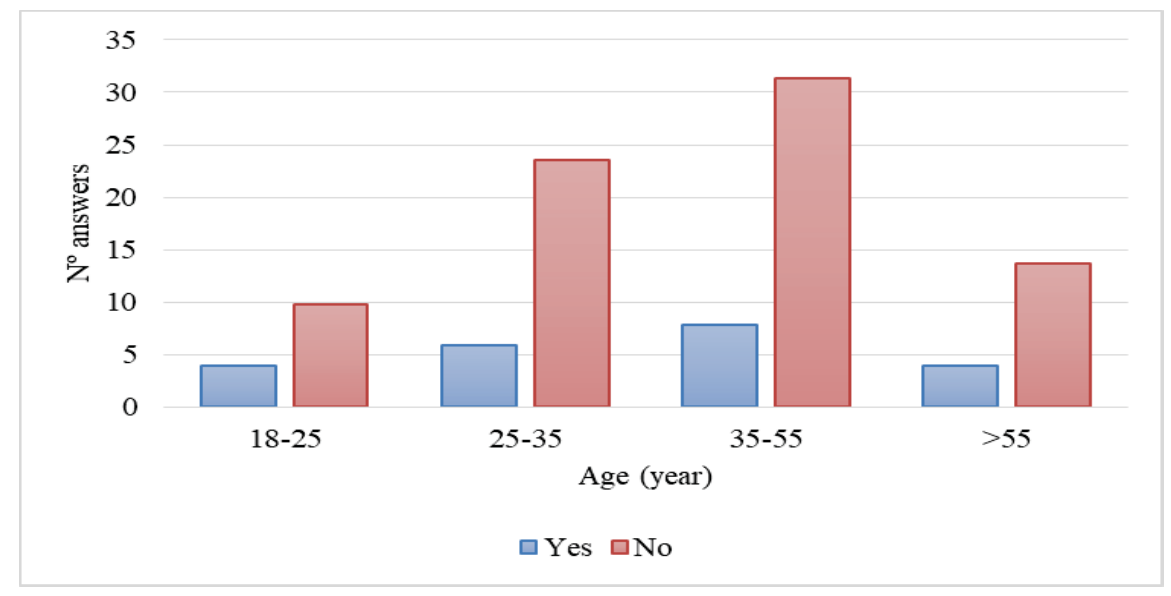

Fig. 3. Is it considered that the rest times are sufficient: yes (blue) and no (orange).

In Fig. 3, 3.92\% of ages between 18 years and 25 years old; $5.88 \%$ of ages between 25 years and 35 years old; $7.84 \%$ between the ages of 35 years and 55 years old; $3.92 \%$ of over 55 years old answered yes (blue); while $9.80 \%$ of ages between 18 years and 25 years old; $23.53 \%$ of ages between 25 years and 35 years old; $31.37 \%$ between the ages of 35 years and 55 years old; $13.73 \%$ of over 55 years old answered no. Summarizing the question presented in Fig. 3, 21.77\% answered yes (blue), while $78.43 \%$ answered no (orange).

Regarding the question is it considered that cumulated tiredness increases the risk of errors: yes (blue) $100 \%$ the distribution by ages is shown in Fig. 4.

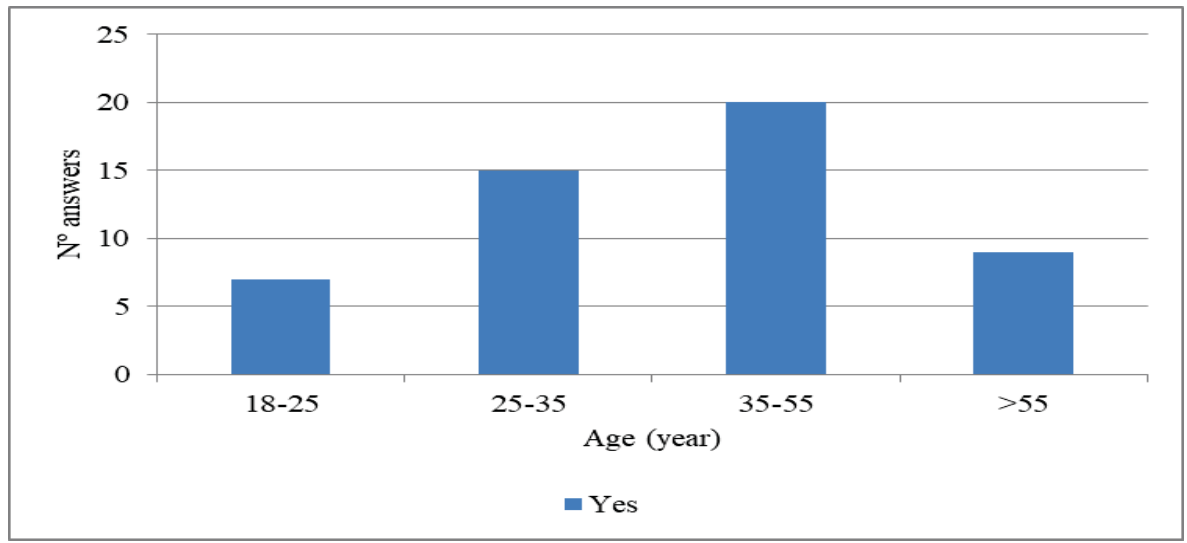

Fig. 4. Is it considered that accumulated tiredness increases the risk of errors: yes (blue). 
In Fig. 4, 13.73\% of ages between 18 years and 25 years old; $29.41 \%$ of ages between 25 years and 35 years old; $39.22 \%$ between the ages of 35 years and 55 years old; $17.65 \%$ of over 55 years' old all answered yes (blue).

Two major concerning facts rise from the survey $[15,16]$. The first is that companies clearly depend on extra hours to carry out all the contracted/required maintenance. Regulations, as previous mentioned, are not clear on this subject, stating only that companies must have the man-power to perform the required assignments, but no additional details are given. The second and most important point is that $95.9 \%$ made or know someone or who made maintenance errors due to stress or fatigue, and from those errors, $17 \%$ were only detected with the aircraft in operation. Also, $55 \%$ of the maintenance errors discovered in operation are made by very experience personal, which $78 \%$ works more than 10 extra hours per week and only $22 \%$ get paid for those extra hours [15].

\section{Hazard procedures}

Aircraft maintenance personnel subject to fatigue, stress or pressure have a negative impact on aircraft safety, once presents the tendency to have hazards behaviours that may led to potential risk of errors [1,2,9,10,15,17-19]. Among the factors related with pressure are the work schedules or hierarchy, which are within the easiest to identify, but are the most difficult to eliminate since are considered inherent to the aviation sector. Some industry players tend to relegate these factors to benefit from manpower cost-saving. When players have this behaviour, it has a clearly negative impact on aircraft safety, as shown in this paper and in $[7,15]$. The maintenance professional's life style also affects their professional performance $[8,20,21]$.

\section{Diagnostic and recommendations}

In [7] authors enumerate the main symptoms of fatigue and stress. The most common symptoms of fatigue are increased reaction time and reduced concentration, diminished memory and mood issues, problems solving routine tasks and been forgetfulness. Regarding the most common symptoms of stress are agitation and frustration, mood issues and depression, having poor judgment and inability to focus.

For the aeronautics industry (operators and maintenance) it is important to build a strong human factors culture oriented to minimize stress, pressure and fatigue by [7,19]: 1) build proper work schedules; 2) provide optimum work conditions; 3) implement team resources management policies together with SMS and Fatigue Risk Management; 4) proper planning to avoid unnecessary overworking or over-planning.

For the aircraft maintenance professionals, it is important to create healthy lifestyle habits according to their daily lifestyle, namely [7,19]: 1) create strict routine of type of meals and sleeping time; 2) monitor the ingestion of caffeine, nicotine and spirits; 3 ) report when on-duty the occurrence of been fatigued, tired or sleepy.

\section{Conclusion}

The conclusion point to circumstances in which regulators should carefully observe the recommendations proposed in this paper. The current regulations are a good starting point, but must be clearer regarding the limits for worktime. Also, a culture driven towards human factors must always be present in all aeronautical actors, without the pressure and stress induced by economic, commercial or time factors. It is clear that Authorities and regulators must regulate the working time periods for the aircraft maintenance professionals like 
already happens with Flight Crew and Air Traffic Controllers. It is also advisable that authorities introduce practical measures to fight the Dirty Dozens more proactively. Maintenance companies and their professionals must be educated how to deal with stress, fatigue and knowing how to identify their symptoms.

\title{
Nomenclature
}

\author{
EASA European Aviation Safety Agency \\ FAA Federal Aviation Administration \\ ICAO International Civil Aviation Organization \\ MOM Maintenance Organization Manual \\ SMS Safety Management System
}

\section{Acknowledgment}

This work is funded by: European Union through the European Regional Development Fund, included in the COMPETE 2020 (Operational Program Competitiveness and Internationalization) through the ICT project UID/GEO/04683/2019 with the reference POCI010145FEDER007690; Portuguese Funds through the Foundation for Science and Technology-FCT under the project LAETA 2015-2020, reference UID/EMS/50022/2019; Portuguese Foundation for Science and Technology (FCT) under Project UID/EMS/00151/2019.

\section{References}

1. ICAO, Safety Management manual (ICAO, 4th ed., Canada, 2018)

2. E. Gerede, Safety Sc. 73, 106-116 (2015)

3. S.M.O. Tavares, P.M.S.T. de Castro, Maintenance, damage tolerance of metallic aircraft structures materials and numerical modelling (17-22, Springer, Heidelberg, 2019)

4. D.A. Wiegmann, S.A. Shappell, A human error approuch to aviation accident analysis (Ashgate Publishing, UK, 2003)

5. M. Ward, N. McDonald, R. Morrison, D. Gaynor, T. Nugent, Ergonomics 53, 2, $247-$ 267 (2010)

6. K.A. Latorella, P.V. Prabhu, Int. J. Ind. Ergonomics 26, 133-161 (2000)

7. L.F.F.M. Santos, R. Melicio, Int. Rev. of Aerosp. Eng. 12, 1, 35-45 (2019)

8. N. Othman, F.I. Romli, Int. Rev. of Aerosp. Eng. 9, 3, 80-84 (2016)

9. ICAO, Annex 19: Safety management (EASA, 2013)

10. A. Hobs, K.B. Avers, J.J. Hils, Fatigue risk management in aviation maintenance: current best practices and potential future countermeasures (1-42, Defense Technical Information Center, Fort Belvoir, Virginia, 2011)

11. EASA, Part-Air Operations (2014)

12. EASA, Part-145, Maintenance organisation approvals (2008)

13. EASA, Part-M, Continuing Airworthiness Requirements (2017)

14. BEA, Final report: accident on 24 March 2015 at Prads-Haute-Bléone, Alpes-deHaute-Provence, France, https://www.bea.aero/uploads/tx_elydbrapports/BEA20150125.en-LR.pdf (2016) 
15. P. Oliveira, Effects of stress and pressure in high performance employments: aeronautical maintenance (Instituto Superior de Educação e Ciências, Lisbon (2017)

16. L.F.F.M. Santos, R. Melicio, Proc. Stakeholders', Meeting on Supply Chain (AERIS), 1-2 (2018)

17. G. Dupont, Proc. 11th Fed. Aviat. Admin. Meet. Human Fact. Iss. Aircraf. Maint. Insp.: Hum. Error Aviat. Maint., 45-49 (1997)

18. FAA AMT Handbook Addendum Human Factors, Federal Aviation Administration (2014)

19. FAA Human Factors Operators Manual, Federal Aviation Administration (2014)

20. J.C. Taylor, R.L. Thomas III, The Int. J. Aviat. Psych. 13, 4, 321-343 (2009)

21. Y.-H. Chang, Y.-Ch. Wang, Saf. Sc. 48, 54-62 (2010) 